\title{
LncRNA MEG3 affects cell proliferation, apoptosis and migration by targeting miR-9-5p/MDK axis and activating PDK/AKT pathway in hepatocellular carcinoma
}

Dezhi Wu ( $\sim$ WuDeZhi1233@163.com )

Zheng Ma

Deyu Ma

Qiquan Li

Research

Keywords: hepatocellular carcinoma, IncRNA MEG3, miR-9-5p, MDK, PDK/AKT pathway

Posted Date: April 8th, 2020

DOI: https://doi.org/10.21203/rs.3.rs-21095/v1

License: (c) (i) This work is licensed under a Creative Commons Attribution 4.0 International License.

Read Full License 


\section{Abstract}

\section{Background}

Long non-coding RNA (IncRNA) maternally expressed gene 3 (MEG3) was supposed to be a tumor suppressor in various cancers. However, the role of MEG3 in hepatocellular carcinoma (HCC) and the related molecular mechanisms are not well illustrated. This study aimed to determine the biological function of MEG3 in regulating HCC cell proliferation, apoptosis and migration. Moreover, the interaction among MEG3, microRNA (miR)-9-5p and Midkine (MDK), and the activation of phosphoinositidedependent kinase (PDK)/AKT pathway in HCC cells were examined.

Methods and Results

Expression of MEG3 in a series of liver cancer cell lines was detected by RT-qPCR. Luciferase reporter assay, RT-qPCR and western blot were used to determine the interaction among MEG3, miR-9-5p and MDK, and the activation of PDK/AKT pathway. Cell proliferation and apoptosis were evaluated by CCK8, flow cytometry analysis for cell cycle and apoptosis, and Caspase $3 / 9$ activity. Cell migration was determined by wound healing assay and MMP1 expression. We found MEG3 was decreased in HCC cell lines compared with the normal liver cell line. MEG3 suppressed HCC cell proliferation and migration, and induced cell apoptosis. Further, we found MEG3 targets miR-9-5p/MDK axis and modulates PDK/AKT pathway in HCC.

Conclusion

Our findings demonstrated that IncRNA MEG3 affects HCC cell proliferation, apoptosis and migration through its targeting of miR-9-5p/MDK and regulating of PDK/AKT pathway. This study suggested MEG3/miR-9-5p/MDK axis as the potential therapeutic target in HCC.

\section{Background}

Hepatocellular carcinoma (HCC), a malignant tumor arising from the liver, accounts for $80 \%$ of primary liver cancer [1]. It is the third leading cause of cancer-related deaths globally [2], and the 5-year survival rate of patients with $\mathrm{HCC}$ is as low as $30 \%$ [3]. Further understanding of hepatocarcinogenesis is imperative, and novel strategies on the treatment of HCC need to be developed.

Long non-coding RNAs (IncRNAs), a class of non-coding transcripts with lengths exceeding 200 nucleotides, participate in a variety of physiological and pathological processes including tumorigenesis [4]. LncRNA maternally expressed gene 3 (MEG3) has been reported to be abnormally expressed in HCC tissues [5, 6], however, its role in HCC and the related mechanisms are not well illustrated. MicroRNAs (miRs) are small ( 22nt) non-coding RNAs which also participate in various biological processes. LncRNAs and miRNAs could interact with each other to serve a wide spectrum of biological functions [7, 
8]. MEG3 has been reported to serve as a competing endogenous RNA (ceRNA) for miR-9-5p in prostate cancer [9] and esophageal cancer [10] to mediate tumor progression.

Midkine (MDK) is a growth factor which initially found in retinoic acid-induced embryonic tumor cells. Currently, MDK is viewed as a multifaceted factor promoting cell growth, survival, metastasis, migration, and angiogenesis, in particular during tumorigenesis [11]. Phosphoinositide-dependent kinase 1 (PDK1) is a serine / threonine protein kinase which plays central roles in signals transduction [12]. Several studies have shown that PDK1 can promote cell proliferation by activating Akt/mTOR signaling pathway [13, 14]. A recent study by Lu et al reported that miR-9 could inhibit angiogenesis by targeting MDK and regulating PDK/AKT pathway in nasopharyngeal carcinoma [15].

In this study, we examined the effect of MEG3 on HCC cell proliferation, apoptosis and migration. Further, we determined the interaction among MEG3, miR-9-5p and MDK, and the activation of PDK/AKT pathway in HCC cells.

\section{Materials And Methods}

\section{Cell culture}

Human normal liver cell line L02 and the liver cancer cell lines (MHCC-97H, MHCC-97L, Hep3B and HepG2) were purchased from Bnbio Company (Beijing, China). The cells were maintained in RPMI 1640 medium (Nanjing KeyGen Biotech. Co. Ltd., Nanjing, Jiangsu, China) supplemented with $10 \%$ fetal bovine serum (FBS; Gibco, Carlsbad, CA, USA), at $37^{\circ} \mathrm{C}$ in a humidified atmosphere of $5 \% \mathrm{CO}_{2}$.

\section{Transfection}

MiR-9-5p mimic, miR-9-5p NC, IncRNA MEG3 overexpression vector and IncRNA MEG3 NC were purchased from General Biosystems, Inc.(Hefei, Anhui,China). Cell transfection was conducted using Lipofectamine 3000 Transfection Reagent (Thermo Fisher Scientific, Waltham, MA, USA) according to the manufacturer's instructions.

\section{Quantitative Real-time Polymerase Chain Reaction (qRT- PCR)}

Total RNA was extracted from the cells using the Ultrapure RNA Kit (CW Biotech, Beijing, China), and then reverse-transcribed into cDNA using a HiFiScriptcDNA Synthesis Kit (CW Biotech). qRT-PCR assays were performed using the UltraSYBR Mixture (CW Biotech). Data were analyzed using the $2-\Delta \Delta$ Ct method.

\section{Western Blot}


Total proteins were extracted, and protein concentrations were quantified via the bicinchoninic acid (BCA) method (Thermo Scientific). A total of $50 \mu \mathrm{g}$ of protein was separated in $10 \%$ SDS-PAGE gels and transferred to PVDF membranes (Millipore, Billerica, MA, USA). After blocking overnight at $4{ }^{\circ} \mathrm{C}$ in the $5 \%$ BSA, the PVDF membranes were incubated with the primary antibodies, including Rabbit Anti AKT (1/500),Rabbit Anti Caspase-3 (1/5000), Rabbit Anti Caspase-9 (1/2000), Rabbit Anti MDK (1/1000), Rabbit Anti MMP-1 (1/1000), Rabbit Anti PDK1(1/2000)(Abcam, Cambridge, UK), Rabbit Anti p-AKT (Bioss, 1/1000), Rabbit Anti p-PDK1 (1/1000;Affinity Biologicals, Ancaster, ON, Canada) and Mouse Monoclonal Anti-GAPDH (1/2000; ZSBIO, Beijing,China) at $4{ }^{\circ} \mathrm{C}$ overnight. Horseradish peroxidase-labeled secondary antibodies, including Goat Anti-Mouse $\lg G(H+L)$ and Goat Anti-Rabbit $\lg G(H+L)(Z S B I O)$, were used at 1:2000 dilution to incubate the membranes at room temperature for $2 \mathrm{~h}$. Signals were detected with the SuperSignal® west pico chemiluminescent substrate (Thermo Fisher Scientific).

\section{Luciferase Reporter Assay}

The wild-type IncRNA MEG3 3'-UTR and MDK 3'-UTR containing the putative binding sites of miR-9-5p were amplified and inserted into the firefly luciferase reporter vector. Cells were co-transfected with miR$\mathrm{NC} / \mathrm{miR}-9-5 \mathrm{p}$ mimic and the wild-type 3'-UTR of MDK or IncRNA MEG3 using Lipofectamine 3000. $48 \mathrm{~h}$ following transfection, firefly and Renilla luciferase activities were detected by the Dual-Luciferase Reporter Assay System (Beyotime, Shanghai, China). The firefly luciferase activity was normalized to the Renilla luciferase activity for each individual analysis.

\section{CCK8}

Cells were seeded at $1 \times 10^{4}$ cells/well in the 96 -well plates. Then, the cells were maintained in RPMI 1640 medium at $37^{\circ} \mathrm{C}$ in an atmosphere of $5 \% \mathrm{CO}_{2}$ for $48 \mathrm{~h}$. Cell viability was measured using a CCK8 kit (Nanjing KeyGen Biotech. Co. Ltd.) according to the manufacturer's instructions. Absorbance at $450 \mathrm{~nm}$ was measured using a Tecan Safire II Microplate Reader (Tecan, Männedorf, Switzerland).

\section{Flow Cytometry (FCM) Analysis Of Cell Cycle}

FCM analysis of cell cycle was performed using a Cell Cycle Staining Kit (MultiSciences(Lianke) Biotech Co., Ltd., Hangzhou, Jiangsu, China) according to the manufacturer's instructions. The cell suspension was centrifuged at $2500 \mathrm{rpm}$ for $3 \mathrm{~min}$, and the cells were then fixed in ethanol at $4{ }^{\circ} \mathrm{C}$ for $2 \mathrm{~h}$. After washing with PBS, $1 \mathrm{ml}$ of DNA staining solution was added to the tubes. The cells were incubated at room temperature in the dark for $30 \mathrm{~min}$, and cell cycle was analyzed by NovoCyte 2060R Flow Cytometer (ACEA Biosciences, Inc., San Diego, CA, USA).

\section{Flow Cytometry Analysis Of Cell Apoptosis}


Cell apoptosis rate was determined by flow cytometry using an Annexin V-FITC/PI Apoptosis Kit (MultiSciences(Lianke) Biotech Co., Ltd.). Briefly, $1 \times 10^{6}$ cells were washed with cold PBS twice, and resuspended in $300 \mu$ l Binding Buffer. After incubation with $3 \mu$ l Annexin V-FITC and $3 \mu$ PI-PE at room temperature for $10 \mathrm{~min}$ in the dark, the cells were mixed with $200 \mu \mathrm{l}$ Binding Buffer, and cell apoptosis rate was analyzed using a NovoCyte 2060R Flow Cytometer (ACEA Biosciences, Inc.).

\section{Wound Healing Assay}

Cells were grown to basically $100 \%$ confluence in 6 -well plates after transfection. The confluent monolayers were scratched by using a 10- $\mu$ l pipette tip, and then washed with PBS for 3 times. Photographs were taken immediately or $24 \mathrm{~h}$ after wounding, and migration was quantified.

\section{Statistical Analyses}

Statistical analyses were performed using SPSS v19.0 (IBM, Armonk, NY, USA). Experimental data were presented as the mean $\pm S D$, and the statistical significance was assessed by oneway analysis of variance. $P<0.05$ was considered significant.

\section{Results}

\section{Expression of IncRNA MEG3 in liver cancer cell lines}

Expression of IncRNA MEG3 in normal liver cell line L02 and liver cancer cell lines (MHCC-97H, MHCC97L, Hep3B and HepG2) were detected by RT-qPCR. As shown in Fig. 1, compared with L02 cell line, the expression of IncRNA MEG3 in MHCC-97H, MHCC-97L, Hep3B and HepG2 cell lines was significantly decreased. Among these liver cancer cell lines, MHCC-97L showed the lowest IncRNA MEG3 expression. We chose MHCC-97L cell line for subsequent experiments.

\section{Effect of IncRNA MEG3 on HCC cell proliferation, apoptosis and migration}

LncRNA MEG3 overexpression vector was transfected into the MHCC-97L cells to investigate the effect of IncRNA MEG3 on HCC cell proliferation, apoptosis and migration. As demonstrated in Fig. 2a, IncRNA MEG3 NC did not affect IncRNA MEG3 expression. Compared with the IncRNA MEG3 NC group, the relative expression of IncRNA MEG3 was significantly increased in the IncRNA MEG3 group $(P<0.01)$. The effect of IncRNA MEG3 on cell proliferation was examined by CCK8 and FCM. The results from CCK8 assay showed that cell viability in the IncRNA MEG3 NC group was decreased significantly when compared with that in the IncRNA MEG3 NC group $(P<0.05$;Fig. $2 b)$. FCM analysis of cell cycle showed that the fraction of S-phase cells was significantly higher and the fraction of G2-phase cells was significantly lower in the IncRNA MEG3 group than in the IncRNA MEG3 NC group $(P<0.05$;Fig. $2 \mathrm{c}$ and 2d).FCM analysis of cell apoptosis revealed that compared with the cells transfected with IncRNA MEG3 
NC, cell apoptosis rate was increased significantly in the cells tansfected with IncRNA MEG3 overexpression vector $(\mathrm{P}<0.05$;Fig. 3a and 3c). Western blot analysis showed that the protein levels of Caspase- 3 and Caspase-9 were upregulated significantly in IncRNA MEG3-transfected cells $(P<0.05$; Fig. 3b). Western blot results (Fig. 4a) indicated that the expression level of MMP1 was evidently lower in the IncRNA MEG3-transfected cells than in the IncRNA MEG3 NC-transfected cells $(P<0.05)$.Wound healing assay was performed to examine the effect of IncRNA MEG3 on cell migration. The results demonstrated that compared with the IncRNA MEG3 NC group, cell migration was inhibited in the IncRNA MEG3 group (Fig. 4b and 4c). LncRNA MEG3 NC did not show any effects on cell proliferation, apoptosis or migration.

\section{LncRNA MEG3 Targets miR-9-5p, And miR-9-5p Targets MDK}

We did luciferase reporter assay to investigate whether IncRNA MEG3 targets miR-9-5p. As shown in Fig. 5a, compared with the IncRNA MEG3 + miR-9-5p mimic NC group, the luciferase activity was decreased significantly in the IncRNA MEG3 + miR-9-5p mimic group $(P<0.05)$. Further, we examined whether MDK was a direct target of miR-9-5p.It was revealed that the luciferase activity was decreased significantly in the MDK + miR-9-5p mimic group when compared with that in the MDK + miR-9-5p mimic $\mathrm{NC}$ group $(\mathrm{P}<0.05)$.

\section{Effect Of IncRNA MEG3 On miR-9-5p And MDK Expression}

The effect of IncRNA MEG3 on miR-9-5p expression was determined by RT-qPCR analysis. As shown in Fig. 5b, compared with the IncRNA MEG3 + miR-9-5p mimic NC group, the relative expression of miR-9-5p was decreased significantly in the IncRNA MEG3 + miR-9-5p mimic group $(P<0.05)$. The effect of IncRNA MEG3 on MDK expression was determined by western blot analysis. We found the relative protein level of MDK was increased significantly in the IncRNA MEG3 + miR-9-5p mimic group when compared with that in the IncRNA MEG3 + miR-9-5p mimic NC group $(P<0.05)$.

\section{Effect Of IncRNA MEG3 On PDK/AKT Signaling}

In order to verify the effect of IncRNA MEG3 on PDK/AKT signaling, western blot assay was performed to examine p-PDK1 and p-AKT expression. Compared with the IncRNA MEG3 NC group, the protein expression of p-PDK1 and p-AKT was upregulated in the IncRNA MEG3 group (both $P<0.05$; Fig. $5 c$ ).

\section{Discussion}

The pathogenesis of HCC is complex, and numerous studies have shown that IncRNAs are involved in the initiation, development and metastasis of HCC [16]. Some studies have shown that MEG3 is significantly 
downregulated in HCC tissues [5, 6]. In agreement with the reports in HCC tissues, we found MEG3 expression is also decreased in a series of HCC cell lines. MHCC-97L cell line showed the lowest expression of MEG3, thus we chose this HCC cell line for the subsequent assays.

Numerous studies reported that restoring MEG3 expression in cancer cells could suppress cell proliferation, invasion as well as angiogenesis, and induce cell apoptosis $[17,18]$. These studies indicated that MEG3 is one of the IncRNAs with tumor suppressor activity [19]. In this study, we transfected MEG3 overexpression vector into MHCC-97L cells to investigate the effect of MEG3 on cell proliferation, apoptosis and migration in HCC. Our results demonstrated that MEG3 inhibits HCC cells proliferation, and a large number of cells accumulated in S phase. Similarly, the result presented by He et al confirmed that MEG3 overexpression suppressed clone formation of hepatoma cells [20].Caspase proteins play important roles in the process of apoptosis. Caspase- 9 is an initiator of apoptosis, and Caspase- 3 is the final executor of apoptosis [21]. MEG3 overexpression led to increased Caspase-3 and Caspase-9 expression, as well as increased cell apoptosis rate, suggesting the promotive role of MEG3 in cell apoptosis. We found MMP1 expression was inhibited by MEG3. MMP1 is involved in invasion and metastasis during the development of cancer, and high expression of MMP1 is associated with poor prognosis of HCC patients [22]. The wound healing assay further verified the inhibitory effect of MEG3 on cell migration. These results were consistent with the previous studies $(5,16)$, and suggested that MEG3 may be a tumor suppressor in HCC.

LncRNAs could serve as a kind of ceRNA to regulate target genes through interacting with miRNAs [23, 24]. Recent studies have clarified that IncRNA-miRNA-mRNA form a novel regulatory network in various pathophysiological processes [25]. The role of MEG3 in cancers has been reported in several studies in which the interaction between IncRNAs, miR and mRNA, has been highlighted [26, 27]. In glioma, MEG3 inhibited the proliferation, migration and invasion of glioma cells by regulating miR-96-5p/MTSS1 [28].In multiple myeloma, MEG3 inhibited tumor progression through regulating the miR-181a/HOXA11 axis [29].Particularly, previous studies have suggested that MEG3/miR-9-5p is involved in regulating the progression of prostate cancer and esophageal cancer by targeting QKI-5 and FOXO1. Recently, a study by Liu et al found that MEG3 can serve as a ceRNA for miR-9-5p to mediate the development of HCC through upregulating SOX11[30].To further explore the molecular mechanisms underlying the role of MEG3 in HCC, we investigated whether MEG3 exerts its protective effects in HCC via regulating miR-9$5 p / M D K$ axis. In this study, to verify the targeting relationship between MEG3, miR-9-5p and MDK, a dual luciferase reporter assay was performed. Consistent with the above reports [9, 30], we found MEG3 directly targets miR-9-5p.Subsequently, we demonstrated that MDK is a direct target of miR-9-5p.This finding was in agreement with a previous study reporting that exosomal miR-9 targets MDK in nasopharyngeal carcinoma [15]. Further, we found the expression of miR-9-5p is downregulated, and the expression of MDK is upregulated by MEG3, indicating that MEG3 could regulate the miR-9-5p/MDK axis in HCC. The PDK/AKT signaling pathway has been suggested to be linked with MDK in angiogenesis, and MDK knockdown led to the inhibition of PDK1 and AKT in HUVEC [15]. In this study, the results revealed that PDK/AKT pathway was activated by MEG3 in HCC. Altogether, these findings demonstrated that MEG3 targets the miR-9-5p/MDK axis and modulates PDK/AKT pathway in HCC. 


\section{Conclusions}

This study demonstrated that MEG3 affects HCC cell proliferation, apoptosis and migration through its targeting of miR-9-5p/MDK and regulating of PDK/AKT pathway. We provided new insights into the molecular mechanisms of MEG3 in HCC, and suggested MEG3/miR-9-5p/MDK axis as the potential therapeutic target in HCC.

\section{Abbreviations}

HCC, hepatocellular carcinoma; IncRNA, long non-coding RNA; MEG3, maternally expressed gene 3; miR, microRNA; MDK, Midkine; PDK, phosphoinositide-dependent kinase.

\section{Declarations}

\section{Acknowledgements}

Not applicable.

\section{Authors' contributions}

DZW and QQL designed the study, analyzed the data and prepared the manuscript. DZW, ZM and DYM conducted the experiments. All authors were substantially

involved in the research, acquisition of data, analysis and manuscript preparation. All authors read and approved the final manuscript.

\section{Funding}

This study was supported by the First-Class Discipline Construction Project in Guizhou Province (Chinese Pharmacy; No.[2017]008).

\section{Availability of data and materials}

The data sets generated and analyzed during the present study are available from the corresponding author on reasonable request.

\section{Ethics approval and consent to participate}

Not applicable.

\section{Consent for publication}

Not applicable.

\section{Conflict of interest}


All authors declare that they have no conflict of interest.

\section{References}

1. McGlynn KA, Petrick JL, London WT. Global epidemiology of hepatocellular carcinoma: an emphasis on demographic and regional variability. Clin Liver Dis. 2015;19:223-8.

2. Siegel RL, Miller KD, Jemal A. Cancer statistics, 2018. CA Cancer J Clin. 2018;68:7-30.

3. 2018 Korean Liver Cancer Association-National Cancer. Center Korea Practice Guidelines for the Management of Hepatocellular Carcinoma. Gut Liver. 2019;13:227-99.

4. Bhan A, Soleimani M, Mandal SS. Long Noncoding RNA and Cancer: A New Paradigm. Cancer Res. 2017;77:3965-81.

5. Zhang Y, Liu J, Lv Y, Zhang C, Guo S. LncRNA meg3 suppresses hepatocellular carcinoma in vitro and vivo studies. Am J Transl Res. 2019;11:4089-99.

6. Zhuo H, Tang J, Lin Z, et al. The aberrant expression of MEG3 regulated by UHRF1 predicts the prognosis of hepatocellular carcinoma. Mol Carcinog. 2016;55:209-19.

7. Lopez-Urrutia E, Bustamante Montes LP, Ladron de Guevara Cervantes D, Perez-Plasencia C, CamposParra AD. Crosstalk Between Long Non-coding RNAs, Micro-RNAs and mRNAs: Deciphering Molecular Mechanisms of Master Regulators in Cancer. Front Oncol. 2019;9:669.

8. Tam C, Wong JH, Tsui SKW, Zuo T, Chan TF, Ng TB. LncRNAs with miRNAs in regulation of gastric, liver, and colorectal cancers: updates in recent years. Appl Microbiol Biotechnol. 2019;103:4649-77.

9. Wu M, Huang Y, Chen T, et al. LncRNA MEG3 inhibits the progression of prostate cancer by modulating miR-9-5p/QKI-5 axis. J Cell Mol Med. 2019;23:29-38.

10. Dong Z, Zhang A, Liu S, et al. Aberrant Methylation-Mediated Silencing of IncRNA MEG3 Functions as a ceRNA in Esophageal Cancer. Mol Cancer Res. 2017;15:800-10.

11. Filippou PS, Karagiannis GS, Constantinidou A. Midkine (MDK) growth factor: a key player in cancer progression and a promising therapeutic target. Oncogene. 2019.

12. Wang F, Shan S, Huo Y, et al. MiR-155-5p inhibits PDK1 and promotes autophagy via the mTOR pathway in cervical cancer. Int J Biochem Cell Biol. 2018;99:91-9.

13. Meng F, Zhang Y, Li X, Wang J, Wang Z. Clinical significance of miR-138 in patients with malignant melanoma through targeting of PDK1 in the PI3K/AKT autophagy signaling pathway. Oncol Rep. 2017;38:1655-62.

14. Chuang C-K, Lin HCA, Tasi H-Y, et al. Clinical presentations and molecular studies of invasive renal epithelioid angiomyolipoma. Int Urol Nephrol. 2017;49:1527-36.

15. Lu J, Liu Q-H, Wang F, et al. Exosomal miR-9 inhibits angiogenesis by targeting MDK and regulating PDK/AKT pathway in nasopharyngeal carcinoma. J Exp Clin Cancer Res. 2018;37:147.

16. Abbastabar M, Sarfi M, Golestani A, Khalili E. IncRNA involvement in hepatocellular carcinoma metastasis and prognosis. EXCLI J. 2018;17:900-13. 
17. Wei GH, Wang X. IncRNA MEG3 inhibit proliferation and metastasis of gastric cancer via p53 signaling pathway. Eur Rev Med Pharmacol Sci. 2017;21:3850-6.

18. Zhang C-Y, Yu M-S, Li X, Zhang Z, Han C-R, Yan B. Overexpression of long non-coding RNA MEG3 suppresses breast cancer cell proliferation, invasion, and angiogenesis through AKT pathway. Tumour Biol. 2017;39:1010428317701311.

19. Zhou Y, Zhang X, Klibanski A. MEG3 noncoding RNA: a tumor suppressor. J Mol Endocrinol. 2012;48:R45-53.

20. He J-H, Han Z-P, Liu J-M, et al. Overexpression of Long Non-Coding RNA MEG3 Inhibits Proliferation of Hepatocellular Carcinoma Huh7 Cells via Negative Modulation of miRNA-664. J Cell Biochem. 2017;118:3713-21.

21. Liu H, Zhou Y, Tang L. Caffeine induces sustained apoptosis of human gastric cancer cells by activating the caspase-9/caspase-3 signalling pathway. Mol Med Rep. 2017;16:2445-54.

22. Altadill A, Rodríguez M, González LO, et al. Liver expression of matrix metalloproteases and their inhibitors in hepatocellular carcinoma. Digestive and liver disease: official journal of the Italian Society of Gastroenterology and the Italian Association for the Study of the Liver. 2009;41: 740-8.

23. Veneziano D, Marceca GP, Di Bella S, Nigita G, Distefano R, Croce CM. Investigating miRNA-IncRNA Interactions: Computational Tools and Resources. Methods in molecular biology (Clifton, N.J.). 2019;1970: $251-77$.

24. Wang X, Su R, Guo Q, Liu J, Ruan B, Wang G. Competing endogenous RNA (ceRNA) hypothetic model based on comprehensive analysis of long non-coding RNA expression in lung adenocarcinoma. PeerJ. 2019;7:e8024.

25. Huang Y. The novel regulatory role of IncRNA-miRNA-mRNA axis in cardiovascular diseases. J Cell Mol Med. 2018;22:5768-75.

26. Zhu D, Xiao Z, Wang Z, et al. MEG3/MIR-376B-3P/HMGA2 axis is involved in pituitary tumor invasiveness. Journal of neurosurgery. 2020: 1-13.

27. Lu WX. Long non-coding RNA MEG3 represses cholangiocarcinoma by regulating miR-3615p/TRAF3 axis. Eur Rev Med Pharmacol Sci. 2019;23:7356-68.

28. Zhang S, Guo W. Long non-coding RNA MEG3 suppresses the growth of glioma cells by regulating the miR-96-5p/MTSS1 signaling pathway. Mol Med Rep. 2019;20:4215-25.

29. Shen $X$, Bai $H$, Zhu $H$, et al. Long Non-Coding RNA MEG3 Functions as a Competing Endogenous RNA to Regulate HOXA11 Expression by Sponging miR-181a in Multiple Myeloma. Cell Physiol Biochem. 2018;49:87-100.

30. Liu Z, Chen JY, Zhong Y, Xie L, Li JS. IncRNA MEG3 inhibits the growth of hepatocellular carcinoma cells by sponging miR-9-5p to upregulate SOX11. Brazilian journal of medical biological research $=$ Revista brasileira de pesquisas medicas e biologicas. 2019;52:e8631.

\section{Figures}



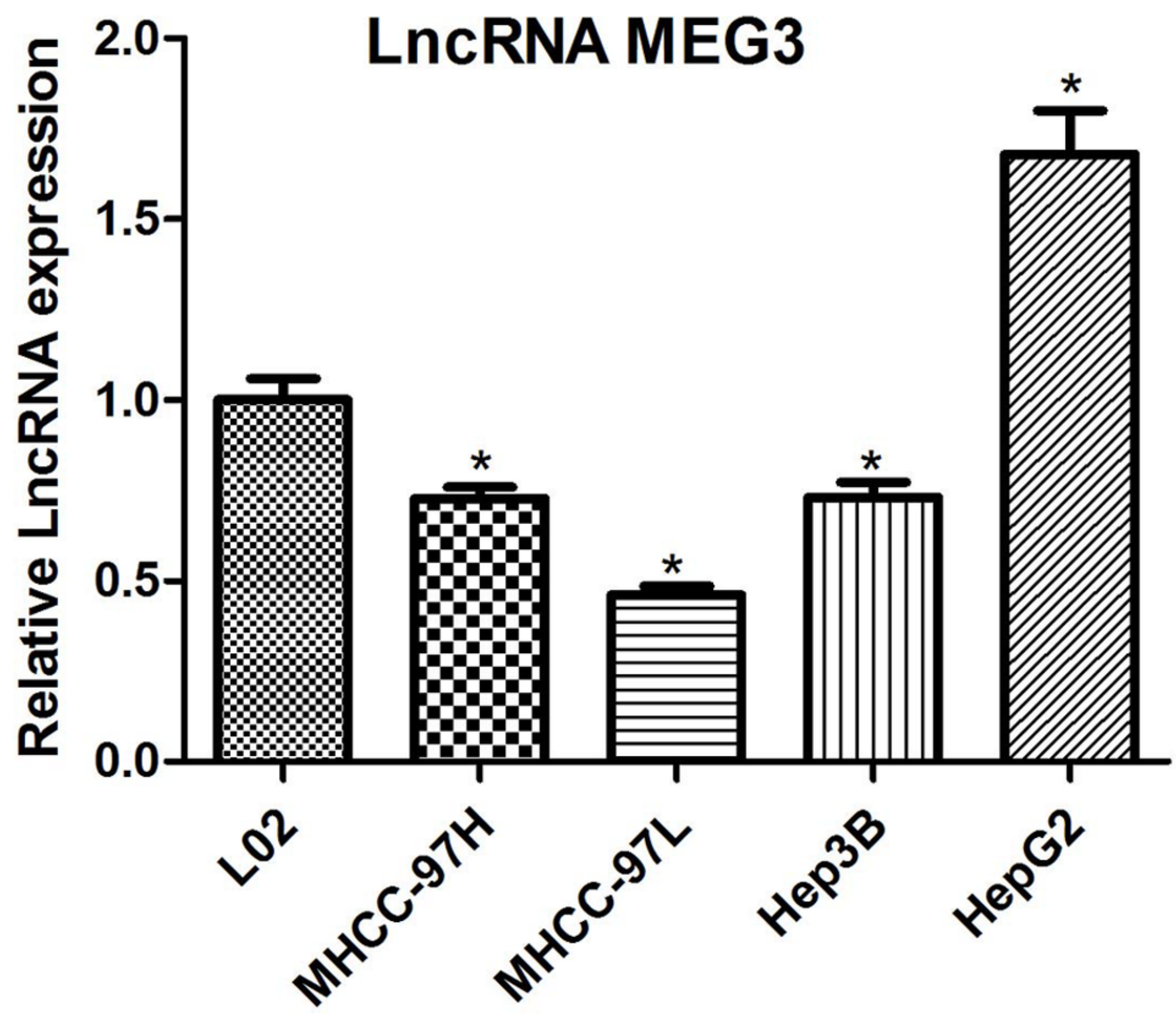

Figure 1

Expression of IncRNA MEG3 in normal liver cell line L02 and liver cancer cell lines (MHCC-97H, MHCC97L, Hep3B and HepG2). ${ }^{\star P}<0.05$ compared with L02.LncRNA MEG3, long non-coding RNA (IncRNA) maternally expressed gene 3 (MEG3). 


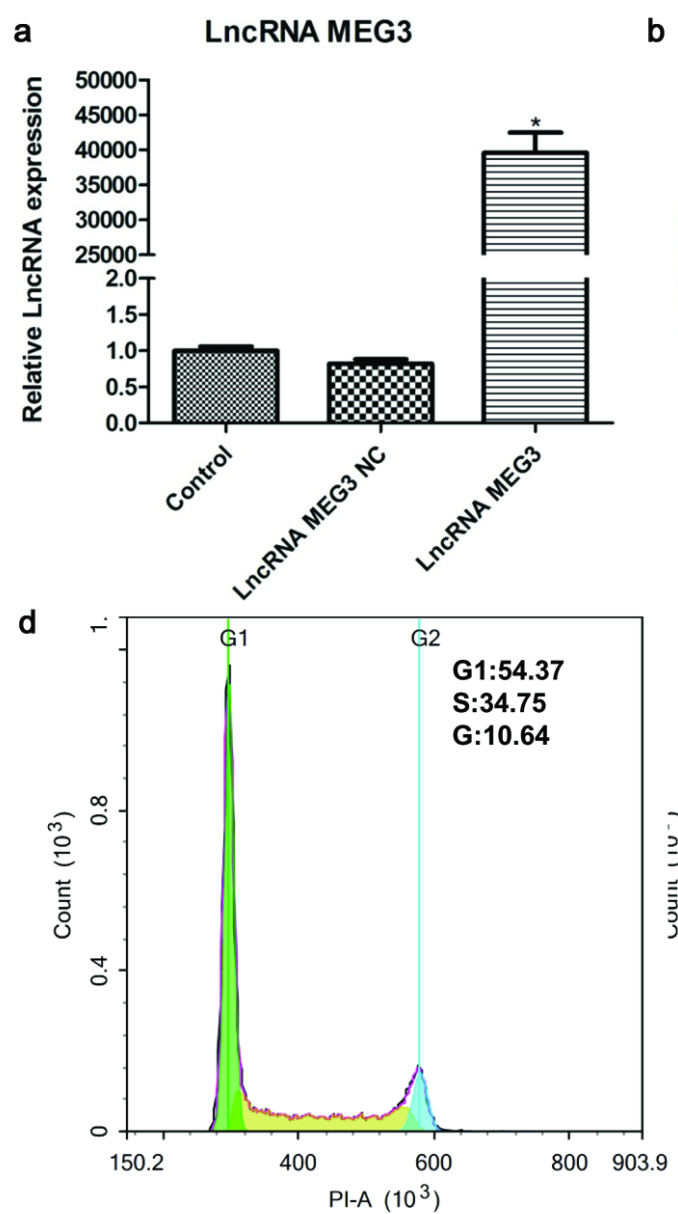

Control

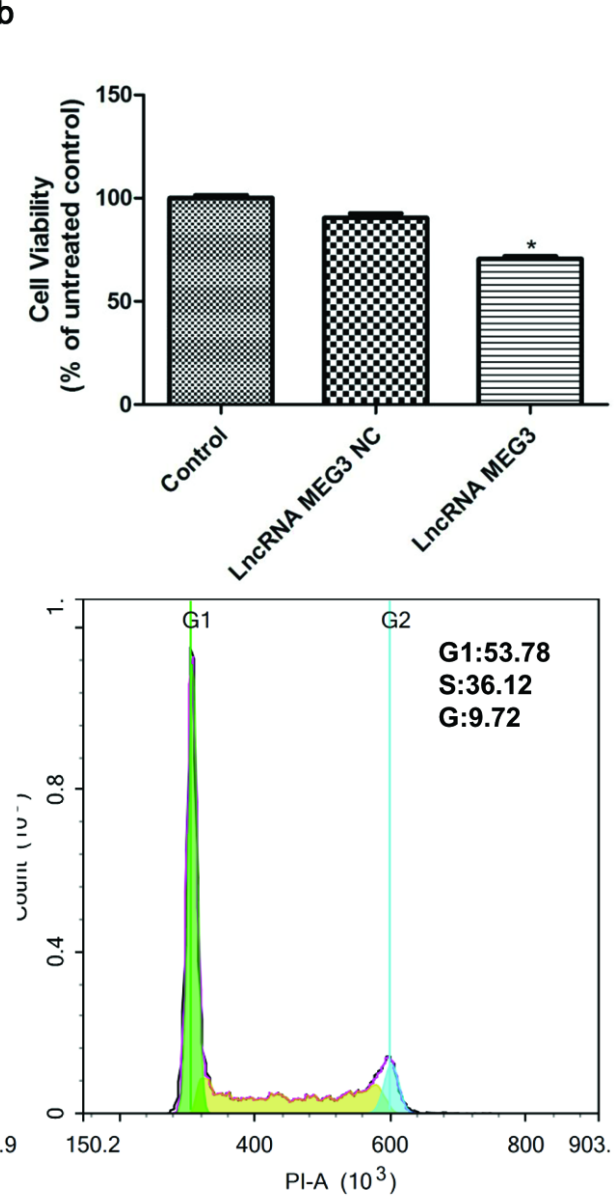

LnCRNA MEG3 NC
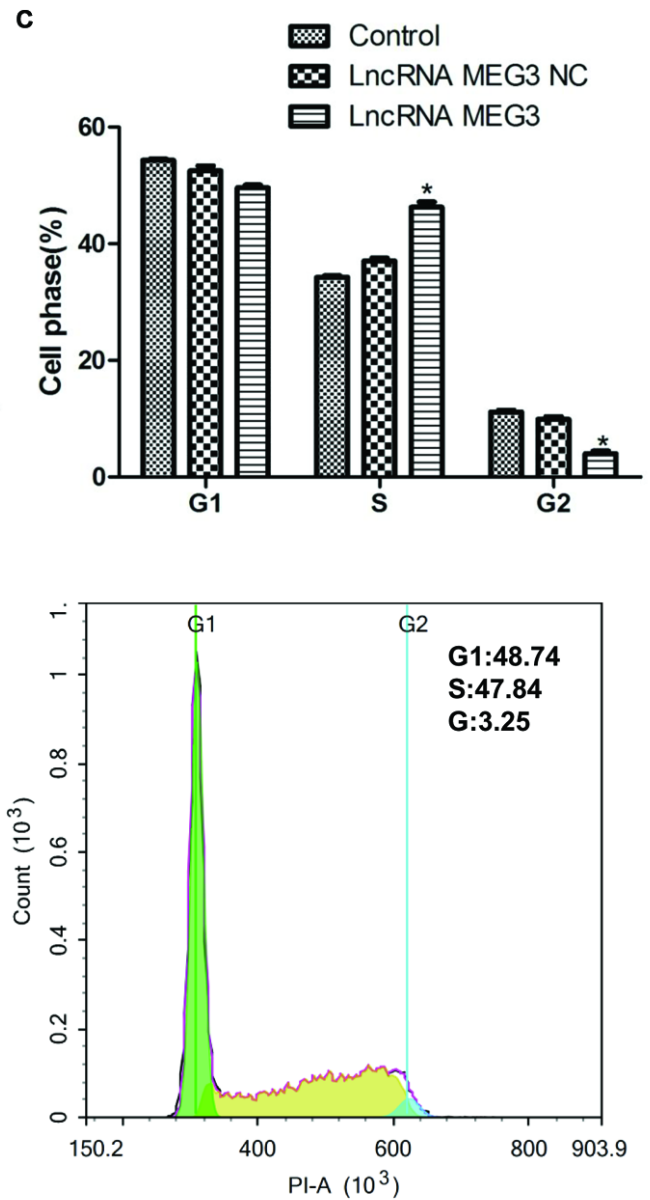

LncRNA MEG3

Figure 2

Effect of IncRNA MEG3 on HCC cell proliferation.(a) The relative expression of IncRNA MEG3 was significantly increased in the cells transfected with the IncRNA MEG3 overexpression vector. ${ }^{*}<0.01$ compared with the LncRNA MEG3 NC group.(b)Cell viability determined by CCK8 assay. ${ }^{*}<0.05$ compared with the LncRNA MEG3 NC group.(c,d) Cell cycle determined by flow cytometry. ${ }^{*} P<0.05$ compared with the LncRNA MEG3 NC group.LncRNA MEG3, long non-coding RNA (IncRNA) maternally expressed gene 3 (MEG3); HCC, hepatocellular carcinoma. 

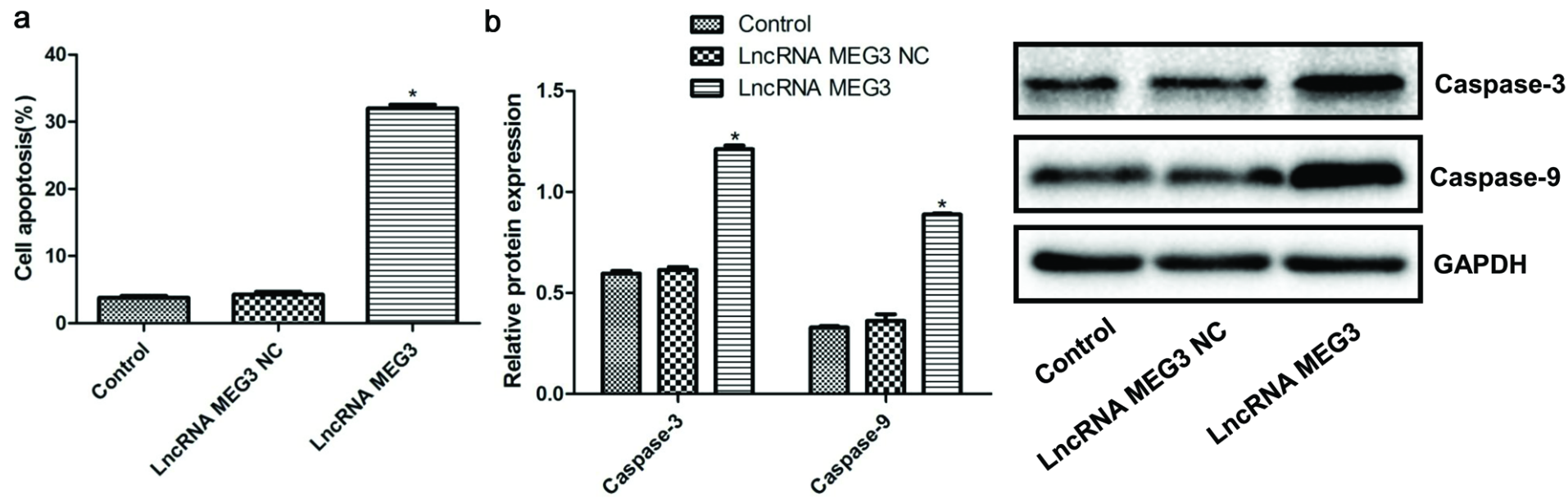

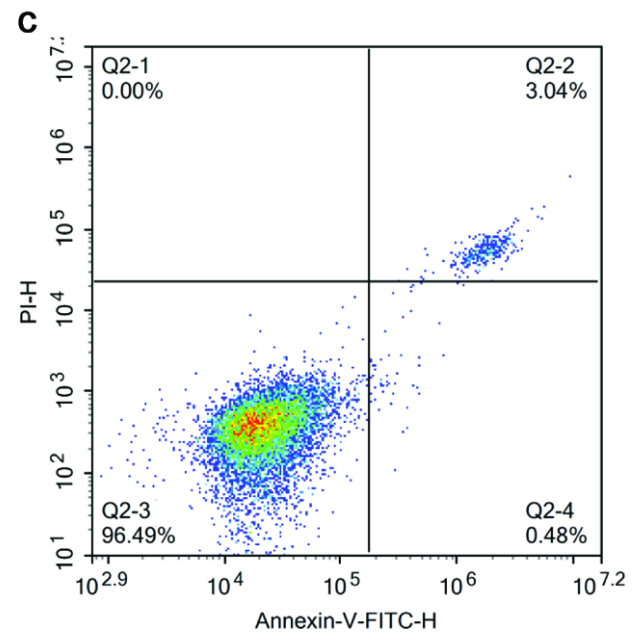

Control

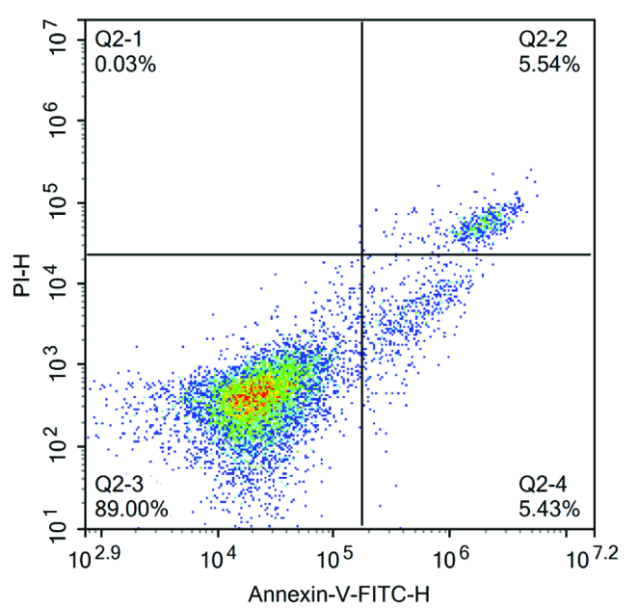

LncRNA MEG3 NC

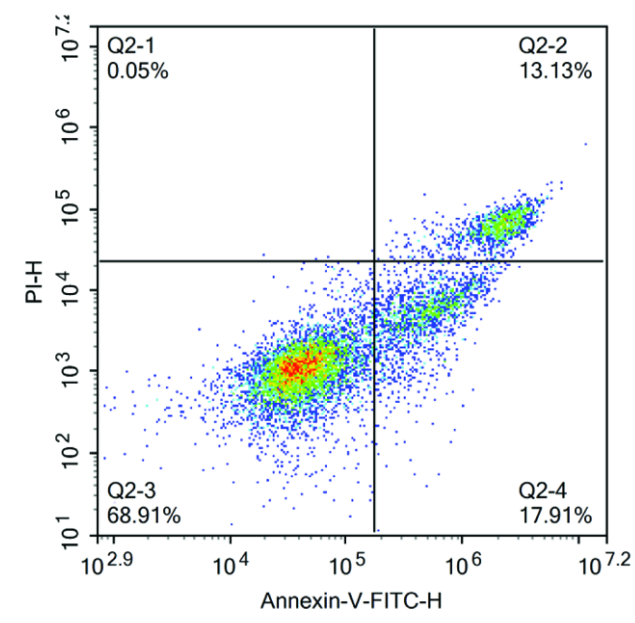

LncRNA MEG3

Figure 3

Effect of IncRNA MEG3 on HCC cell apoptosis.(a,c)Cell apoptosis rate determined by flow cytometry. ${ }^{*} \mathrm{P}<0.01$ compared with the LncRNA MEG3 NC group.(b)Relative protein expression of Caspase-3 and Caspase- 9 determined by western blot. ${ }^{\star} \mathrm{P}<0.05$ compared with the LncRNA MEG3 NC group.LncRNA MEG3, long non-coding RNA (IncRNA) maternally expressed gene 3 (MEG3); HCC, hepatocellular carcinoma. 


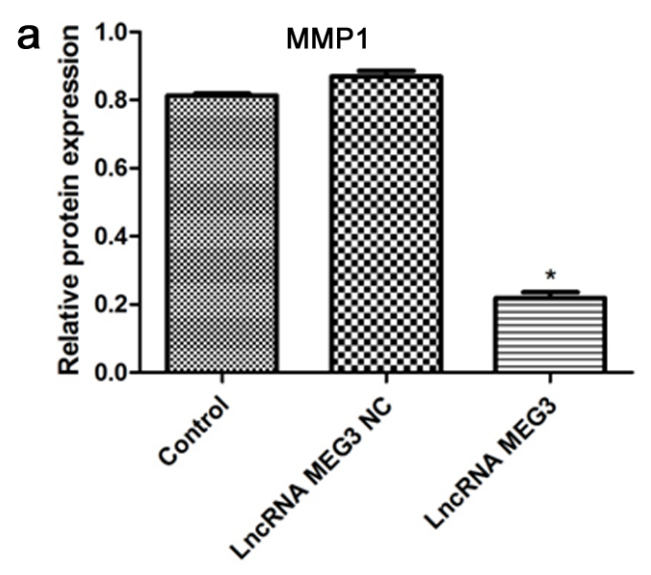

C
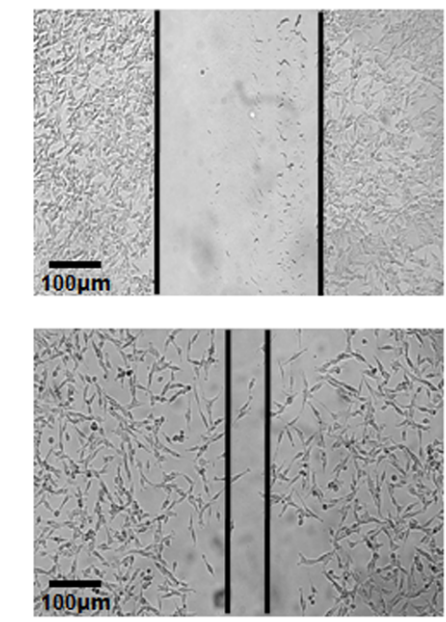

Control
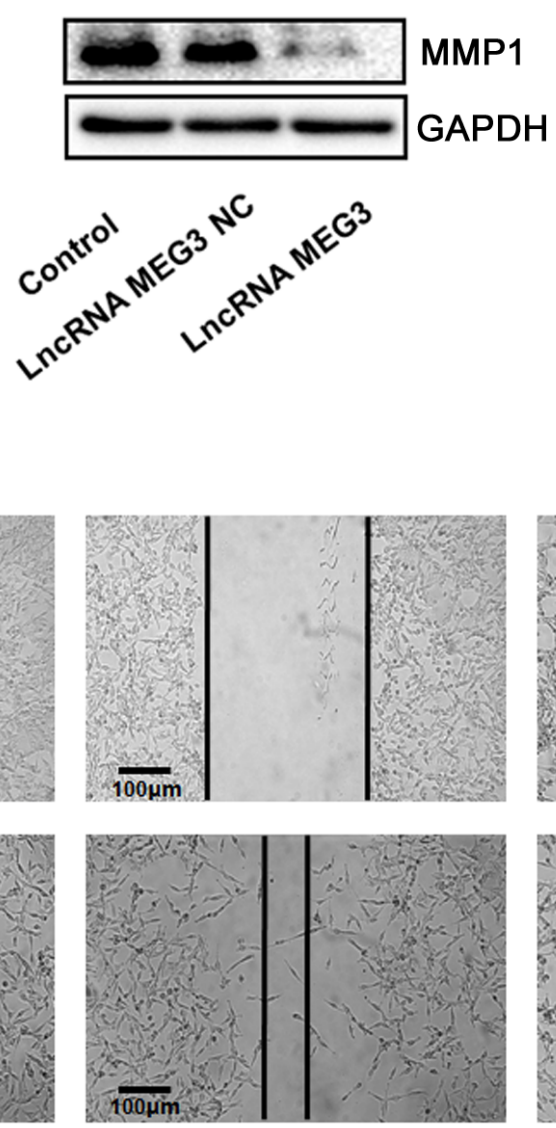

LnCRNA MEG3 NC b
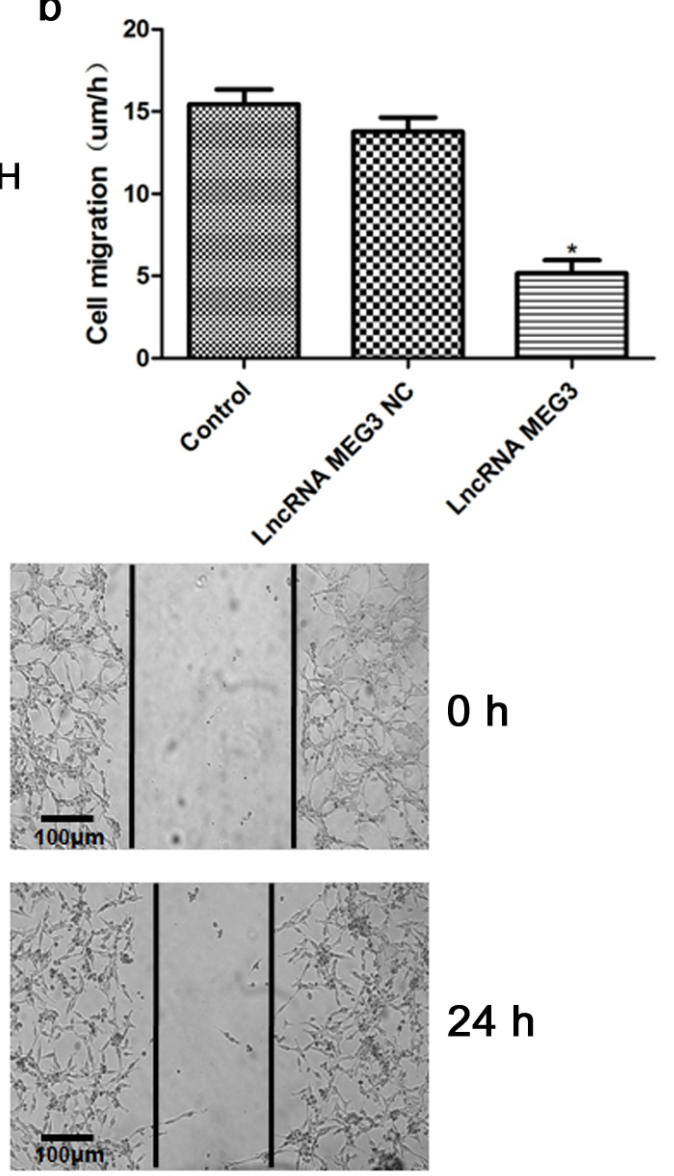

LncRNA MEG3

\section{Figure 4}

Effect of IncRNA MEG3 on HCC cell migration.(a)Relative protein expression of MMP1 determined by western blot. ${ }^{*} P<0.05$ compared with the LncRNA MEG3 NC group. $(b, c)$ Cell migration determined by wound healing assay. ${ }^{*}<0.05$ compared with the LncRNA MEG3 NC group. LncRNA MEG3, long noncoding RNA (IncRNA) maternally expressed gene 3 (MEG3); HCC, hepatocellular carcinoma. 
a
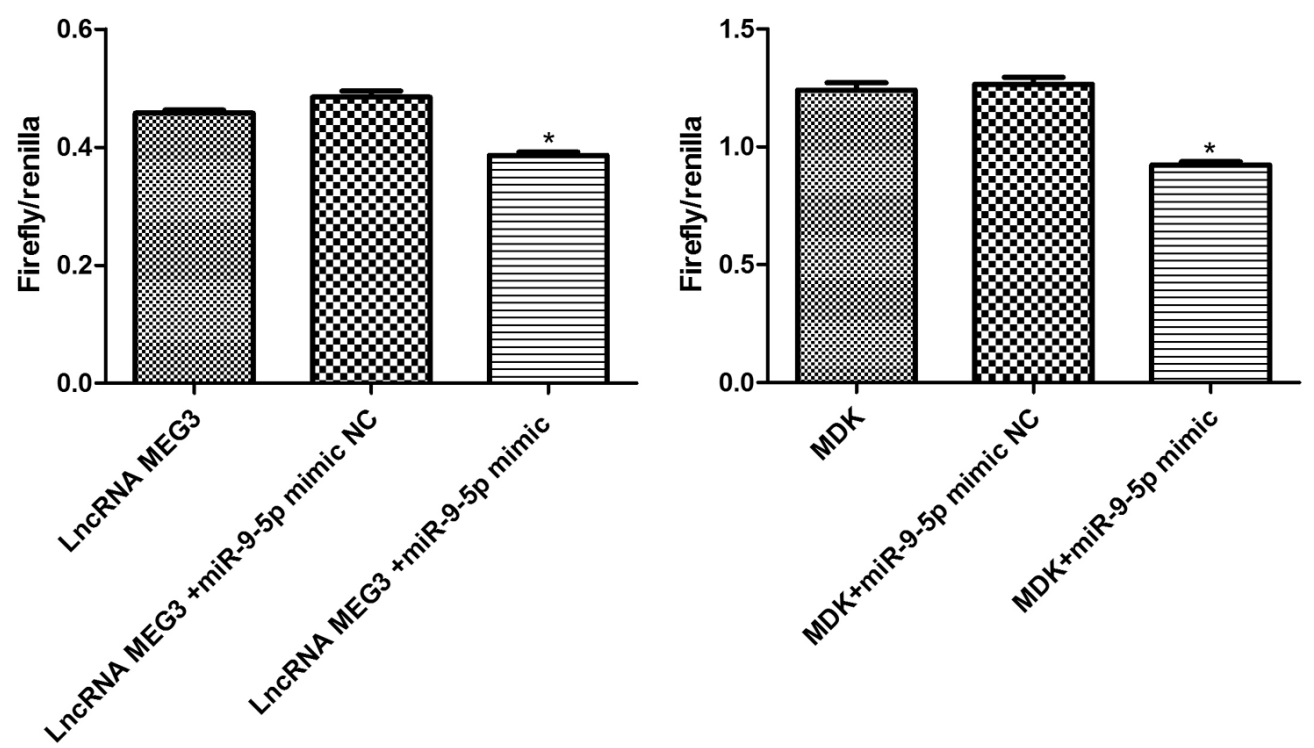

b
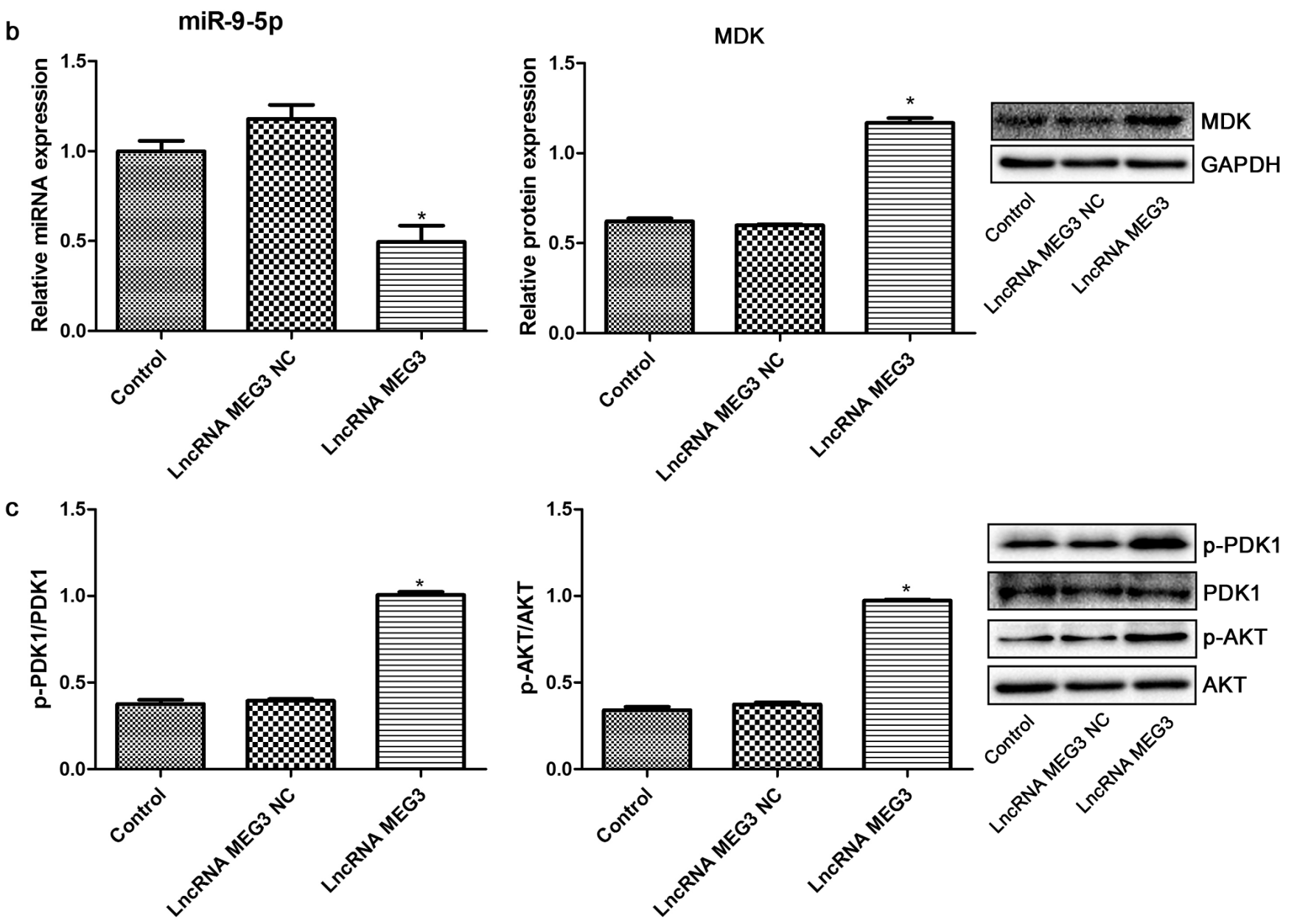

Figure 5

The interaction among MEG3, miR-9-5p and MDK, and the effect of MEG3 on PDK/AKT pathway activation.(a)LncRNA MEG3 targets miR-9-5p ( ${ }^{*} \mathrm{P}<0.05$ compared with the LncRNA MEG3+miR-9-5p mimic NC group), and miR-9-5p targets MDK ( ${ }^{*}<<0.05$ compared with the MDK+miR-9-5p mimic NC group), as determined by luciferase reporter assay.(b) Effect of IncRNA MEG3 on miR-9-5p and MDK expression. ${ }^{*}<0.05$ compared with the LncRNA MEG3 NC group.(c) Effect of IncRNA MEG3 on PDK/AKT 
signaling. ${ }^{*}<<0.05$ compared with the LncRNA MEG3 NC group. LncRNA MEG3, long non-coding RNA (IncRNA) maternally expressed gene 3 (MEG3); miR, microRNA; MDK, Midkine; PDK1, phosphoinositidedependent kinase 1. 